\title{
How does Extrusion Technology Help the Development of Foods with Better Nutritional Value?
}

\author{
Jose Luis Ramirez Ascheri1*, Ronel Joel Bazan Colque², Laysa Borges Tomas De Sousa ${ }^{3}$, Diego Palmiro \\ Ramirez Ascheri ${ }^{4}$ and Erika Madeira Moreira Da Silva ${ }^{5}$
}

${ }^{1}$ Researcher at Embrapa Food Technology, Food Extrusion and Physical Properties Lab, Rio de Janeiro, RJ, Brazil

${ }^{2}$ Federal Rural University of Rio de Janeiro (UFRRJ)

${ }^{3,5}$ Federal University of Espirito Santo- (UFES), Brazil

${ }^{4}$ Postgraduate of Agricultural Engineering Course, Campus of Exact and Technological Sciences, Brazil

*Corresponding author: Jose Luis Ramirez Ascheri, Researcher at Embrapa Food Technology, Food Extrusion and Physical Properties Lab, Brazil.

Received Date: January 07, 2019

Published Date: February 06, 2019

\begin{abstract}
Extrusion is nowadays a technological procedure that allows the processing of a very large quantity of final products. These may be for human, animal or industrial use. Food for human consumption has been a major focus in the use of this technological process. Because of their versatility of use, there is a possibility of using very different raw materials, which individually or mixed with other products can generate high added value food products. In this work we point out that only in the adequate control of the process parameters can we obtain foods of high nutritional value, with good sensory characteristics and functional properties.
\end{abstract}

Keywords: Extrusion cooking; Products Development; Parameters processing; Quality criteria; Foods; Nutrition values

\section{Introduction}

First, let us define what extrusion is. There are several definitions in this regard, one of which describes as a thermomechanical process of high temperature and short time (HTST), which combines moisture, heat and mechanical shear. That by transporting, by means of a screw or screws inside a barrel, at a high rotation speed, restricted by a die or several dies, the material is modified deeply creating new forms and structures with different physical, chemical, nutritional and sensorial properties [1]. The question is that, the equipment, raw materials, working conditions in the plant, are in convergent way to the good norms and practices of operation and quality. Good results in the physical, functional and sensorial properties require the good management of all the parameters involved, from the adequate and excellent quality of the raw material as in the management of extrusion system parameters including moisture processing, barrel temperature, screw speed, suitable pre-conditioning system, and feeding system. Obviously due care after extrusion: time and temperature of drying, proper packaging and storage.

\section{Extrusion-Cooking as A Tool for New Products}

Extrusion technology, by operational versatility and its multiple functions, has a wide range of applications, whether in the alimentary area for human or animal consumption, or in the industrial area (manufacture of plastic packaging, foam fillings, for drilling of oil wells, etc.). By this technique, a great variety of products can be obtained, the main ones being mentioned below:

Foodstuffs for human consumption: baking area: cookies, pasta, cracker cookies, wafers, porridges, etc. cereal and snack area: Ready-to-eat cereals, fortified cereals. Snacks, pellets, ingredients for other foods.

Let's look at an example of how extrusion can contribute to the nutritional quality of a particular product, in this case the production of instant cereal baby porridge. Considering corn grits, it would be preferable to use QPM corn grits or its equivalent in essential amino acids (lysine, tryptophan and methionine), rice, to consider the one with higher levels of minerals; especially Zc and Fe, fortified are 
available. Sorghum flour may be included in the mix, or according to the criteria of the formulator and its consumer target, the use of other cereals or even some legumes, [2]. These possibilities are related to the use of people with celiac disease, limiting itself in this case to discarding cereals that contain gluten [3]. Once the mixture is in the correct proportions with cereals and/or leguminous flours, the baby food can be processed, and in order not to lose too much nutrients, the parameter adjustment is very important. Excess temperature, speed of screw beyond limits, cause the product to undergo major transformations in the chemical structure. About $30 \%$ of the amino acid quality can be lost [4]. Of course, larger transformations also entail different physical functional properties, in this case, absorption index and water solubility, pasting properties with very low values for a porridge. In some cases, the formulations may be supplemented with bioactive agents, such as essential amino acids important in human nutrition, vitamins and minerals. This way you can enrich the final product and give greater value to the product. As an example of this practice of enrichment with amino acids, minerals and other bioactive compounds, we put the animal feed industry for poultry, shrimp, among other species. These additions are fundamental because they are the only food source for these species. Therefore, that productive sector obtains records of production by its nutritional quality obtained through the extrusion. Another important event to consider, in which extrusion technology contributes and can still exploit much more, is the use of byproducts resulting from agricultural exploitation. It is known that millions of tons of coproducts are thrown away [5]. Many of these interfere with the environment quality. This is because there are many factors that prevent them from being exploited: little interest in adding value, due to low knowledge, thinking is a few environmental damages, so there is not much obligation to transformations these wastes. Other aspect is lack of investment and public policies to take this issue seriously. Many works, [6] mainly academic ones, have been developed, with the intention to give alternatives of use to the by-products [7] discarded of the industry. An example, it may be mentioned, is the use of the passion fruit peel associated with cereals, such as rice, allowing the mixed flour resulting from extrusion processing to be used as a carrier of dietary fiber. That is one of the serious questions in the feeding of the populations of the big metropolis, by the low consumption of fibers [3]. In the same way, since it is not possible or not easy for the population to accept in nature grapefruit peel flour, in this sense its association with extruded rice flour makes it feasible to use this material in the preparation of other foods according to their need or imagination in the form of consumption. In both cases, there are some gains in these new products, the possibility of being ingested higher contents of antioxidants, in this case, present in the grapes peel. Obviously, it will not be with the total composition of its original grape peel, but even processed there are still significant levels of antioxidants in the products processed by extrusion, [810].

\section{Conclusion}

In conclusion, the production of precooked foods by extrusion is an important alternative for application to different raw materials, so that when it is not possible to process in a certain condition, it can be assisted with the use of other farinaceous ingredients with adequate rates with rice flour corn grits, flour sorghum, among other cereals and grains, according to their regional availability.

\section{Acknowledgement}

The authors would like to thank them for research stimulation grant to FAPERJ, FAPES, CAPES and CNPq for their financial support.

\section{Conflict of Interest}

No Conflict of Interest.

\section{References}

1. Vivian Offiah, Vassilis Kontogiorgos, Kolawole O, Falade (2018) Extrusion Processing of Raw Food Materials and by-products: A Review. Critical Reviews in Food Science and Nutrition.

2. Anuonye JC, Jigam AA, Yndaako GM (2012) Effects of extrusion cooking on the nutrients and antinutrients composition of pigeon pea and unripe plantain blends. Journal of Applied Pharmaceutical Science 2(5): 58-162.

3. Silva, Erika Madeira Moreira da, Ascheri, Jose Luis Ramirez, Ascheri, et al. (2016) Quality assessment of gluten-free pasta prepared with a brown rice and corn meal blend via thermoplastic extrusion. LWT-Food Science and Technology 68: 698 - 706.

4. Camire ME, Camire A, Krumhar K (1990) Chemical and nutritional changes in foods during extrusion. Crit. Rev Food Sci Nutr 29: 35-57.

5. Ascheri, Diego Palmiro Ramirez, Cavalcante LH, Ascheri Jose Luis Ramirez, Carvalho CWP (2016) Physical-chemical characterization of pre-cooked mixed rice flour and barley bagasse. Semina Ciencias Agrarias 37: 750 .

6. Walter Willet (2019) Food in the Anthropocene: the EAT-Lancet Commission on healthy diets from sustainable food systems.

7. Mohamed Elleuch, Dorothea Bedigian, Olivier Roiseux, Souhail Besbes, Christophe Blecker, et al. (2011) Dietary fiber and fiber-rich by-products of food processing: Characterisation, technological functionality and commercial applications: A review. Food Chemistry 124: 411-421.

8. Teba, Carla Da Silva, Silva, Erika Madeira Moreira Da, Chavez, et al. (2017) Effects of whey protein concentrate, feed moisture and temperature on the physicochemical characteristics of a rice-based extruded flour. Food Chemistry 228: 287-296.

9. Chavez, Davy WH, Ascheri, Jose LR, Carvalho, et al. (2017) Sorghum and roasted coffee blends as a novel extruded product: Bioactive compounds and antioxidant capacity. Journal of Functional Foods 29: 93 - 103.

10. Khavhatondwi Rinah Netshiheni, Mpho Edward Mashau, Afam Israel Obiefuna Jideani (2018) Nutritional and sensory properties of instant maize porridge fortified with Moringa oleifera leaves and termite (Macrotermes falciger) powders. Nutrition \& Food Science. 